The original publication of this paper contains errors. The correct Table 1 is shown in this paper.

\title{
Reptile surveys reveal high species richness in areas recovering from mining activity in the Brazilian Cerrado
}

\author{
Fabrício H. OdA ${ }^{1,2}$, Robson W. Ávila ${ }^{3}$, Leandro de O. Drummond ${ }^{4}$, Danusy L. Santos ${ }^{5}$,
} Priscilla G. Gambale ${ }^{5,6}$, Vinicius Guerra ${ }^{5,6}$, Raísa Romęnia S. VieIRA ${ }^{7}$, Tiago S. Vasconcelos ${ }^{8}$, Rogério P. Bastos $^{5} \&$ Fausto Nomura $^{5}$

${ }^{1}$ Centro Universitário Cesumar, Programa de Pós-Graduação em Promoção da Saúde, 87050-390, Maringá, Paraná, Brazil; e-mails: fabricio_oda@hotmail.com,fabricio.oda@gmail.com

${ }^{2}$ Laboratório de Ictioparasitologia, Núcleo de Pesquisas em Limnologia, Ictiologia e Aqüicultura, Universidade Estadual de Maringá, 87020-900, Maringá, Paraná, Brazil

${ }^{3}$ Universidade Regional do Cariri, Departamento de Cięncias Biológicas, 63105-100, Crato, Ceará, Brazil

${ }^{4}$ Universidade Federal do Rio de Janeiro, Programa de Pós-Graduação em Ecologia, 21941-902, Rio de Janeiro, Rio de Janeiro, Brazil

${ }^{5}$ Universidade Federal de Goiás, Departamento de Ecologia, Instituto de Cięncias Biológicas, Laboratório de Herpetologia e Comportamento Animal, Goiânia, Goiás, Brazil

${ }^{6}$ Universidade Estadual de Maringá, Programa de Pós-graduação em Ecologia de Ambientes Aquáticos Continentais, 87020900, Maringá, Paraná, Brazil

${ }^{7}$ Universidade Federal de Goiás, Departamento de Ecologia, Instituto de Ciências Biológicas, Laboratório de Biogeografia da Conservação, 74001-970, Goiânia, Goiás, Brazil

${ }^{8}$ Universidade Estadual Paulista, Departamento de Ciências Biológicas, Faculdade de Ciências, 17033-360 Bauru, São Paulo, Brazil

Table 1. Locations within the Cerrado domain with comparison of its reptile assemblages and the respective record for the municipality of Niquelândia, Goiás state, central Brazil. Only squamata reptile species were considered in the analyses.

\begin{tabular}{|c|c|c|c|c|c|c|c|c|}
\hline $\mathrm{N}$ & Location & Ns & $\mathrm{Ch}$ & $\mathrm{Cr}$ & $\mathrm{Lz}$ & $\mathrm{Am}$ & Sn & Source \\
\hline 1 & Serra do Gado Bravo, MA & 29 & 2 & - & 12 & 2 & 13 & Barreto et al. (2007) \\
\hline 2 & Paquetá farm, PI & 31 & 1 & 1 & 14 & 1 & 14 & Silva et al. (2015) \\
\hline 3 & Uruçuí-Una Ecological Station, PI & 64 & 1 & 1 & 19 & 4 & 39 & Dal Vechio et al. (2013) \\
\hline 4 & Serra das Confusões National Park, PI & 47 & 2 & - & 21 & 4 & 20 & Dal Vechio et al. (2016) \\
\hline 5 & $\begin{array}{l}\text { Grande Sertão Veredas National Park, } \\
\text { BA/MG }\end{array}$ & 50 & - & - & 18 & 2 & 9 & Recoder \& Nogueira (2007) \\
\hline 6 & $\begin{array}{l}\text { Campo das Vertentes and Serra das Ver- } \\
\text { tentes, MG }\end{array}$ & 31 & 2 & - & 9 & 2 & 18 & Sousa et al. (2010) \\
\hline 7 & Unilavras-Boqueirão Biological Reserve, MG & 17 & - & - & 11 & - & 6 & $\begin{array}{l}\text { Novelli et al. (2012), Lucas et al. } \\
(2016)\end{array}$ \\
\hline 8 & Serra do Ouro Branco, MG & 43 & - & - & 15 & - & 28 & $\begin{array}{l}\text { São Pedro \& Pires (2009), } \\
\text { Rosário Cruz et al. (2014) }\end{array}$ \\
\hline 9 & Serra de São José, MG & 29 & - & - & 12 & 1 & 16 & Rios et al. (2017) \\
\hline 10 & Batalha Hydroeletric Power Plant, MG/GO & 54 & 2 & 1 & 17 & 2 & 32 & Lagos et al. (2017) \\
\hline 11 & $\begin{array}{l}\text { Assis Ecological Station and municipality of } \\
\text { Assis, SP }\end{array}$ & 53 & - & - & 10 & 4 & 39 & Araujo \& Almeida-Santos (2011) \\
\hline 12 & Aporé-Sucuriú Complex, MS/SP/MG/GO & 36 & - & 1 & 17 & 4 & 14 & Uetanabaro et al. (2006) \\
\hline 13 & Serra da Bodoquena National Park, MS & 25 & 1 & 1 & 15 & 1 & 7 & Uetanabaro et al. (2007) \\
\hline 14 & $\begin{array}{l}\text { Ponte de Pedra Hydroeletric Power Plant, } \\
\text { MT/MS }\end{array}$ & 72 & 2 & 1 & 23 & 8 & 40 & Silva Jr. et al. (2009) \\
\hline 15 & Middle Aporé River, MS/GO & 33 & - & 1 & 14 & - & 18 & Ramalho et al. (2014) \\
\hline 16 & $\begin{array}{l}\text { Meandros do rio Araguaia Environmental } \\
\text { Protection Area, GO/MT }\end{array}$ & 28 & - & - & 13 & - & 15 & Santos et al. (2008) \\
\hline 17 & Manso Hydroeletric Power Plant region, MT & 78 & 2 & 2 & 26 & 4 & 44 & Strüssmann (2000) \\
\hline 18 & Entre Rios Hydroelectric Power Plant, MT & 24 & 1 & - & 12 & - & 11 & Silva et al. (2015) \\
\hline 19 & Municipality of Alto Araguaia, MT & 13 & 1 & - & 8 & - & 4 & Mendes-Pinto \& Miranda (2011) \\
\hline
\end{tabular}


Table 1. (continued)

\begin{tabular}{|c|c|c|c|c|c|c|c|c|}
\hline $\mathrm{N}$ & Location & Ns & $\mathrm{Ch}$ & $\mathrm{Cr}$ & $\mathrm{Lz}$ & Am & Sn & Source \\
\hline 20 & Municipality of Nobres, MT & 28 & - & - & 13 & 1 & 14 & Santos et al. (2011) \\
\hline 21 & $\begin{array}{l}\text { Santa Edwiges I and II Hydroeletric Power } \\
\text { Plant, GO }\end{array}$ & 45 & - & - & 14 & 3 & 28 & Cintra et al. (2009) \\
\hline 22 & Southeastern Goiás state, GO & 40 & 2 & 1 & 13 & 3 & 21 & Santos et al. (2014) \\
\hline 23 & National Forest Silvânia, GO & 32 & 1 & - & 14 & - & 17 & Morais et al. (2012) \\
\hline 24 & Upper Tocantins River, GO & 47 & - & - & 13 & 7 & 27 & Moreira et al. (2009) \\
\hline 25 & Emas National Park, GO & 87 & - & - & 27 & 6 & 54 & Valdujo et al. (2009) \\
\hline 26 & Espora Hydroeletric Power Plant, GO & 71 & 2 & 1 & 18 & 5 & 45 & Vaz-Silva et al. (2007) \\
\hline 27 & $\begin{array}{l}\text { Areas recovering and municipality of } \\
\text { Niquelândia, GO }\end{array}$ & 47 & 1 & 1 & 17 & 2 & 26 & This study \\
\hline 28 & $\begin{array}{l}\text { Luís Eduardo Magalhães Hydroeletric Power } \\
\text { Plant, TO }\end{array}$ & 111 & 6 & 2 & 26 & 7 & 70 & Pavan \& Dixon (2004) \\
\hline 29 & Jalapão region, TO & 88 & 1 & 2 & 27 & 6 & 52 & $\begin{array}{l}\text { Vitt et al. (2005), Recoder et al. } \\
\text { (2011) }\end{array}$ \\
\hline 30 & Águas Emendadas Ecological Station, DF & 52 & 2 & 2 & 17 & 2 & 29 & Brandão \& Araújo (1998) \\
\hline 31 & $\begin{array}{l}\text { Cafuringa Environmental Protection Area, } \\
\text { DF }\end{array}$ & 48 & 3 & - & 19 & 2 & 24 & Brandão et al. (2006) \\
\hline 32 & IBGE Biological Reserve, DF & 63 & 1 & - & 20 & 5 & 37 & Colli et al. (2011) \\
\hline
\end{tabular}

Abbreviation: Ns - number of species, Ch - Chelonian, Cr - Crocodilian, Lz - Lizard, Am - Amphisbaenian, Sn - Snake. 